\title{
Current Single Event Effects Compendium of Candidate Spacecraft Electronics for NASA
}

\author{
Martha V. O'Bryan, Kenneth A. LaBel, Jonathan A. Pellish, Dakai Chen, Jean-Marie Lauenstein, \\ Cheryl J. Marshall, Ray L. Ladbury, Timothy R. Oldham, Hak S. Kim, Anthony M. Phan, \\ Melanie D. Berg, Martin A. Carts, Anthony B. Sanders, Stephen P. Buchner, Paul W. Marshall, \\ Michael A. Xapsos, Farokh Irom, Larry G. Pearce, Eric T. Thomson, Theju M. Bernard, \\ Harold W. Satterfield, Alan P. Williams, Nick W. van Vonno, James F. Salzman, \\ Sam Burns, and Rafi S. Albarian
}

\begin{abstract}
We present the results of single event effects (SEE) testing and analysis investigating the effects of radiation on electronics. This paper is a summary of test results.
\end{abstract}

Index Terms-Single event effects, spacecraft electronics, digital, linear bipolar, and hybrid devices.

\section{INTRODUCTION}

The performance of electronic devices in a space radiation environment is often limited by its susceptibility to SEE. Interpreting the results of SEE testing of complex devices is quite difficult. As discussed elsewhere [1], SEE test data is often application specific and adequate understanding of the test conditions is critical.

This work was supported in part by the NASA Electronic Parts and Packaging Program (NEPP), NASA Flight Projects, the Defense Threat Reduction Agency (DTRA) under IACRO\# 09-4587I and 10-4977I, NASA/GSFC Internal Research \& Development Program, and the Space Radiation Element Human Research program at NASA/JSC.

Martha V. O'Bryan is with MEI Technologies Inc., c/o NASA Goddard Space Flight Center (GSFC), Code 561.4, Bldg. 22, Rm. 062A, Greenbelt, MD 20771 (USA), phone: 301-286-1412, fax: 301-286-4699, email: martha.v.obryan@nasa.gov.

Kenneth A. LaBel, Jonathan A. Pellish, Jean-Marie Lauenstein, Cheryl Marshall, Ray L. Ladbury, Martin A. Carts, Anthony B. Sanders, and Michael A. Xapsos are with NASA/GSFC, Code 561.4, Greenbelt, MD 20771 (USA), phone: 301-286-9936 (LaBel), 301-286-6523 (Pellish), 301-286-5587 (Lauenstein), 434-376-3402 (Marshall), 301-286-1030 (Ladbury), 301-2862600 (Carts), 301-286-8046 (Sanders), 301-286-2263 (Xapsos), email: kenneth.a.label@nasa.gov, jonathan.a.pellish@nasa.gov, jean.m.lauenstein@ nasa.gov, cheryl.j.marshall@nasa.gov, raymond.1.ladbury@nasa.gov, martin .a.carts@nasa.gov, anthony.b.sanders@nasa.gov, and michael.a.xapsos@ nasa.gov.

Dakai Chen, Hak S. Kim, Anthony M. Phan, and Melanie Berg are with MEI Technologies, Inc., c/o NASA/GSFC, Code 561.4, Greenbelt, MD 20771 (USA), phone: 301-286-1023 (Kim), 301-286-1239 (Phan), 301-286-8595 (Chen), 301-286-2153 (Berg), email: Hak.S.Kim@ nasa.gov, Anthony.M.Phan @ nasa.gov, Dakai.Chen-1@ nasa.gov and Melanie.D.Berg @ nasa.gov.

Timothy R. Oldham is with Perot Systems Government Services, Inc., c/o NASA/GSFC, Code 561.4, Greenbelt, MD 20771 (USA), phone: 301-2865489, email: timothy.r.oldham@ nasa.gov.

Stephen P. Buchner is with Global Defense Technology and Systems, Inc., phone: 202-404-2352, email: stephen.buchner.ctr@nrl.navy.mil.

Paul Marshall is a Consultant, email: pwmarshall@aol.com.

Farokh Irom is with JPL, email: farokh.irom@jpl.nasa.gov.

Larry G. Pearce, Eric T. Thomson, Theju M. Bernard, Harold W. Satterfield, Alan P. Williams, and Nick W. van Vonno, are with Intersil Corporation, Palm Bay, Florida, email: nvv@cfl.rr.com.

James F. Salzman is with Texas Instrument, email: salzman@ti.com.

Sam Burns, Rafi S. Albarian are from Linear Technology, emails: sburns@linear.com, ralbarian@linear.com.
Given this limitation of test data (application-specific), studies discussed here were undertaken to establish the sensitivities of candidate spacecraft electronics as well as new electronic devices to heavy ion and proton-induced single event upset (SEU), single event latchup (SEL), and single event transients (SET). For total ionizing dose (TID) and displacement damage results, see a companion paper submitted to the 2010 IEEE NSREC Radiation Effects Data Workshop entitled: "Current Total Ionizing Dose and Displacement Damage Compendium of Candidate Spacecraft Electronics for NASA" by D. Cochran, et al. [2].

\section{TeSt TeChNIQUES AND SETUP}

\section{A. Test Facilities}

All SEE tests were performed between February 2009 and February 2010. Heavy ion experiments were conducted at Lawrence Berkeley National Laboratory (LBNL) [3], and at Texas A\&M University Cyclotron (TAMU) [4]. Both of these facilities are suitable for providing a variety of ions over a range of energies for testing. The devices under test (DUTs) were irradiated with heavy ions having linear energy transfers (LETs) ranging from 0.59 to $120 \mathrm{MeV} \cdot \mathrm{cm}^{2} / \mathrm{mg}$. Fluxes ranged from $1 \times 10^{2}$ to $1 \times 10^{7}$ particles $/ \mathrm{cm}^{2} / \mathrm{s}$, depending on device sensitivity. Representative ions used are listed in Table I. LETs between the values listed were obtained by changing the angle of incidence of the ion beam with respect to the DUT, thus changing the path length of the ion through the DUT and the "effective LET" of the ion [5]. Energies and LETs available varied slightly from one test date to another.

Proton SEE tests were performed at three facilities: the University of California at Davis (UCD) Crocker Nuclear Laboratory (CNL) [6], the Indiana University Cyclotron Facility (IUCF) [7], and at a $2 \mathrm{MeV}$ Van de Graaff particle accelerator. Proton test energies incident on the DUT are listed in Table II.

Laser SEE tests were performed at the pulsed laser facility at the Naval Research Laboratory (NRL) [8] [9]. The laser light had a wavelength of $590 \mathrm{~nm}$ resulting in a skin depth (depth at which the light intensity decreased to $1 / \mathrm{e}$ - or about $37 \%$ - of its intensity at the surface) of $2 \mu \mathrm{m}$. A nominal pulse rate of $1 \mathrm{kHz}$ was utilized. 
TABle I: Heavy Ion Test Facilities And Test Heavy Ions

\begin{tabular}{|c|c|c|c|c|}
\hline & Ion & $\begin{array}{c}\text { Energy } \\
\text { (MeV) }\end{array}$ & $\begin{array}{c}\text { Surface } \\
\text { LET in Si } \\
\left(\mathrm{MeV} \cdot \mathrm{cm}^{2} / \mathrm{mg}\right) \\
\text { (Normal Incidence) }\end{array}$ & $\begin{array}{c}\text { Range in } \\
\text { Si }(\mu \mathrm{m})\end{array}$ \\
\hline \multirow[t]{7}{*}{ LBNL } & ${ }^{18} \mathrm{O}$ & 184 & 2.2 & 227 \\
\hline & ${ }^{22} \mathrm{Ne}$ & 216 & 3.5 & 175 \\
\hline & ${ }^{40} \mathrm{Ar}$ & 400 & 9.7 & 130 \\
\hline & ${ }^{65} \mathrm{Cu}$ & 659 & 21 & 110 \\
\hline & ${ }^{86} \mathrm{Kr}$ & 886 & 31 & 110 \\
\hline & ${ }^{136} \mathrm{Xe}$ & 1330 & 59 & 97 \\
\hline & \multicolumn{4}{|c|}{$10 \mathrm{MeV}$ per AMU tune } \\
\hline \multirow[t]{12}{*}{ TAMU } & ${ }^{20} \mathrm{Ne}$ & 300 & 2.5 & 316 \\
\hline & ${ }^{40} \mathrm{Ar}$ & 599 & 7.7 & 229 \\
\hline & ${ }^{63} \mathrm{Cu}$ & 944 & 17.8 & 172 \\
\hline & ${ }^{84} \mathrm{Kr}$ & 1259 & 25.4 & 170 \\
\hline & ${ }^{109} \mathrm{Ag}$ & 1634 & 38.5 & 156 \\
\hline & ${ }^{129} \mathrm{Xe}$ & 1934 & 47.3 & 156 \\
\hline & \multicolumn{4}{|c|}{$15 \mathrm{MeV}$ per AMU tune } \\
\hline & ${ }^{22} \mathrm{Ne}$ & 545 & 1.8 & 799 \\
\hline & ${ }^{40} \mathrm{Ar}$ & 991 & 5.5 & 493 \\
\hline & ${ }^{84} \mathrm{Kr}$ & 2081 & 19.8 & 332 \\
\hline & ${ }^{139} \mathrm{Xe}$ & 3197 & 38.9 & 286 \\
\hline & \multicolumn{4}{|c|}{$25 \mathrm{MeV}$ per AMU tune } \\
\hline
\end{tabular}

TABLE II: PRoton TEST FACILITIES

University of California at Davis (UCD) Crocker Nuclear Laboratory (CNL), energy tunes ranged from 6.5 to $63 \mathrm{MeV}$, flux ranged from $8 \times 10^{7}$ to $1 \times 10^{9}$ particles $/ \mathrm{cm}^{2} / \mathrm{s}$.

Indiana University Cyclotron Facility (IUCF), energy ranged from 63 to $198 \mathrm{MeV}$, flux ranged from $5 \times 10^{5}$ to $3 \times 10^{9} \mathrm{particles} / \mathrm{cm}^{2} / \mathrm{s}$.

TABLE III: LASER TEST FACILITY

Naval Research Laboratory (NRL) Pulsed Laser SEE Test Facility

Laser: $590 \mathrm{~nm}, 1$ ps pulse width, beam spot size $\sim 1.2 \mu \mathrm{m}$

\section{B. Test Method}

Unless otherwise noted, all tests were performed at room temperature and with nominal power supply voltages. We recognize that high-temperature and worst-case power supply conditions are recommended for single event latchup (SEL) device qualification.

\section{1) SEE Testing - Heavy Ion:}

Depending on the DUT and the test objectives, one or more of three SEE test methods were typically used:

Dynamic - the DUT was exercised continually while being exposed to the beam. The events and/or bit errors were counted, generally by comparing the DUT output to an unirradiated reference device or other expected output (Golden chip or virtual Golden chip methods) [10]. In some cases, the effects of clock speed or device operating modes were investigated. Results of such tests should be applied with caution due to the application-specific nature of the results.

Static - the DUT was loaded prior to irradiation; data were retrieved and errors were counted after irradiation.
Biased - the DUT was biased and clocked while power consumption was monitored for SEL or other destructive effects. In most SEL tests, functionality was also monitored.

In SEE experiments, DUTs were monitored for soft errors, such as SEUs and for hard errors, such as single event gate rupture (SEGR). Detailed descriptions of the types of errors observed are noted in the individual test results [11].

SET testing was performed using a high-speed oscilloscope. Individual criteria for SETs are specific to the device being tested. Please see the individual test reports for details [11].

Heavy ion SEE sensitivity experiments include measurement of the Linear Energy Transfer threshold (LET $\left.{ }_{\text {th }}\right)$ and cross section at the maximum measured LET. The $\mathrm{LET}_{\mathrm{th}}$ is defined as the maximum LET value at which no effect was observed at an effective fluence of $1 \times 10^{7}$ particles $/ \mathrm{cm}^{2}$. In the case where events are observed at the smallest LET tested, LET $_{\text {th }}$ will either be reported as less than the lowest measured LET or determined approximately as the $\mathrm{LET}_{\text {th }}$ parameter from a Weibull fit. In the case of SEGR experiments, measurements are made of the SEGR threshold $V_{d s}$ as a function of LET at a fixed $\mathrm{V}_{\mathrm{gs}}$.

\section{2) SEE Testing - Proton}

Proton SEE tests were performed in a manner similar to heavy ion exposures. However, because protons cause SEE via indirect ionization of recoil particles, results are parameterized in terms of proton energy rather than LET. Because such proton-induced nuclear interactions are rare, proton tests also feature higher cumulative fluences and particle flux rates than heavy ion experiments.

\section{3) Pulsed Laser Facility Testing}

The DUT was mounted on an X-Y-Z stage in front of a $100 \mathrm{x}$ lens that produced a spot size of about $1.2 \mu \mathrm{m}$ at fullwidth half-maximum (FWHM). The X-Y-Z stage can be moved in steps of $0.1 \mu \mathrm{m}$ for accurate positioning of SEU sensitive regions in front of the focused beam. An illuminator together with a charge coupled device camera and monitor were used to image the area of interest, thereby facilitating accurate positioning of the device in the beam. The pulse energy was varied in a continuous manner using a polarizer/half-waveplate combination and the energy was monitored by splitting off a portion of the beam and directing it at a calibrated energy meter.

\section{Test Results OVERVIEW}

Abbreviations and conventions are listed in Table IV. Abbreviations for principal investigators (PIs) are listed in Table V, and SEE results are summarized in Table VI. Unless otherwise noted, all LETs are in $\mathrm{MeV} \cdot \mathrm{cm}^{2} / \mathrm{mg}$ and all cross sections are in $\mathrm{cm}^{2} /$ device. This paper is a summary of results. Complete test reports are available online at http://radhome.gsfc.nasa.gov [11]. 
TABLE IV: ABBREVIATIONS AND CONVENTIONS

LET $=$ linear energy transfer $\left(\mathrm{MeV} \cdot \mathrm{cm}^{2} / \mathrm{mg}\right)$

$\mathrm{LET}_{\mathrm{th}}=$ linear energy transfer threshold (the minimum LET value for which a given effect is observed for a fluence of $1 \times 10^{7}$ particles $/ \mathrm{cm}^{2}$ - in $\mathrm{MeV} \cdot \mathrm{cm}^{2} / \mathrm{mg}$ )

$<=$ SEE observed at lowest tested LET

$>=$ no SEE observed at highest tested LET

$\sigma=$ cross section $\left(\mathrm{cm}^{2} /\right.$ device, unless specified as $\left.\mathrm{cm}^{2} / \mathrm{bit}\right)$

$\sigma_{\max }$ measured $=$ cross section at maximum measured LET $\left(\mathrm{cm}^{2} /\right.$ device, unless specified as $\left.\mathrm{cm}^{2} / \mathrm{bit}\right)$

$\mathrm{ADC}=$ analog to digital converter

App. Spec. $=$ application specific

BiCMOS = bipolar complementary metal oxide semiconductor

CMOS = complementary metal oxide semiconductor

DUT $=$ device under test

EDAC $=$ error detection and correction

FPGA = field programmable gate array

$\mathrm{H}=$ heavy ion test

$\mathrm{L}=$ laser test

LCDT $=$ low cost digital tester

$\mathrm{LDC}=$ lot date code

$\mathrm{N} / \mathrm{A}=$ not available

$\mathrm{P}=$ proton test $(\mathrm{SEE})$

$\mathrm{PI}=$ principal investigator

$\mathrm{POL}=$ point of load

$\mathrm{SEE}=$ single event effect

SEFI = single event functional interrupt

SEL $=$ single event latchup

SET $=$ single event transient

SEU $=$ single event upset

$\mathrm{SiGe}=$ silicon germanium

SEGR = single event gate rupture

$\mathrm{V}_{\mathrm{ds}}=$ drain-source voltage

$\mathrm{V}_{\mathrm{gs}}=$ gate-source voltage
TABLE V: LIST OF PRINCIPAL INVESTIGATORS

\begin{tabular}{lc}
\hline \multicolumn{1}{c}{ Principal Investigator (PI) } & Abbreviation \\
\hline Melanie Berg & $\mathrm{MB}$ \\
Martin Carts & $\mathrm{MaC}$ \\
Dakai Chen & $\mathrm{DC}$ \\
Hak Kim & $\mathrm{HK}$ \\
Kenneth LaBel & $\mathrm{KL}$ \\
Ray Ladbury & $\mathrm{RL}$ \\
Jean-Marie Lauenstein & $\mathrm{JML}$ \\
Cheryl Marshall & $\mathrm{CM}$ \\
Paul Marshall & $\mathrm{PM}$ \\
Timothy Oldham & $\mathrm{TO}$ \\
Jonathan Pellish & $\mathrm{JP}$ \\
Anthony (Tony) Sanders & $\mathrm{AS}$ \\
Michael Xapsos & $\mathrm{MX}$ \\
\hline
\end{tabular}

TABLE VI: Summary of SEE TeSt Results

\begin{tabular}{|c|c|c|c|c|c|c|c|c|c|c|}
\hline Part Number & Manufacturer & LDC & $\begin{array}{l}\text { Device } \\
\text { Function }\end{array}$ & $\begin{array}{l}\text { Tech- } \\
\text { nology }\end{array}$ & $\begin{array}{c}\text { Particle: } \\
\text { (Facility/Date) P.I., }\end{array}$ & $\begin{array}{l}\text { Test Results LET in } \\
{\mathrm{MeV} \cdot \mathrm{cm}^{2} / \mathrm{mg} \sigma \text { in }} \mathrm{cm}^{2} / \mathrm{device} \text {, unless } \\
\text { otherwise specified }\end{array}$ & 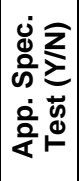 & $\begin{array}{l}\text { 총 巳 } \\
\text { 을 } \\
\text { ஸे }\end{array}$ & 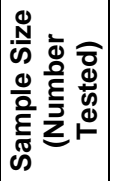 & 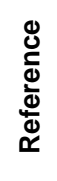 \\
\hline \multicolumn{11}{|l|}{ ADC : } \\
\hline ADC14155 & $\begin{array}{l}\text { National } \\
\text { Semi- } \\
\text { conductor }\end{array}$ & $\begin{array}{l}\text { No LDC } \\
\text { (test chip) }\end{array}$ & ADC & CMOS & $\begin{array}{l}\text { P: (UCD09JUN) } \\
\text { MB }\end{array}$ & $\begin{array}{l}\text { Low energy proton testing to } \\
\text { investigate direct ionization. } \\
\text { Errors were observed at } \\
\text { proton energies near } 1 \mathrm{MeV} \\
\text { and above; All low energy } \\
\text { errors were analog (no digital } \\
\text { errors detected); Potential } \\
\text { direct ionization was } \\
\text { observed. } \\
\end{array}$ & $\mathrm{N}$ & $5 \mathrm{~V}$ & 2 & $\begin{array}{l}{[12]} \\
{[13]}\end{array}$ \\
\hline ADS5483 & $\begin{array}{c}\text { Texas } \\
\text { Instrument }\end{array}$ & $\begin{array}{c}\text { delidded } \\
\text { by TI } \\
\text { markings: } \\
\text { AZ5483 } \\
\text { TI } 83 \mathrm{~K} \\
\text { E7NO G } \\
\end{array}$ & ADC & $\begin{array}{c}\text { Comple } \\
\text { mentary } \\
\text { Bipolar } \\
\text { (BiCom3) }\end{array}$ & $\begin{array}{l}\text { H: (TAMU09MAY) } \\
\text { MB (w/Robert } \\
\text { MacDowell) } \\
\text { P: (IU09AUG) MB }\end{array}$ & $\begin{array}{l}\mathrm{H}: \mathrm{LET}_{\text {th }}<8.6 \text { (no LET SEE); } \\
\text { SEL LET th }>75 \\
\mathrm{P}: \mathrm{SEE} \sigma \text { ranged from } 2 \times 10^{-10} \\
\mathrm{~cm}^{2} / \text { device at } 75 \mathrm{MeV} \text { to } \\
2.5 \times 10^{-10} \mathrm{~cm}^{2} / \text { device at } \\
198 \mathrm{MeV} .\end{array}$ & $\mathrm{N}$ & $\begin{array}{l}3.3 \mathrm{~V} \\
5 \mathrm{~V}\end{array}$ & $\begin{array}{l}\mathrm{H}: 2 \\
\mathrm{P}: 4\end{array}$ & $\begin{array}{l}{[13]} \\
{[14]}\end{array}$ \\
\hline \multicolumn{11}{|l|}{ FPGA: } \\
\hline RTAX2000S & Actel & 0526 & $\begin{array}{l}\text { RTAX-S } \\
\text { FPGA }\end{array}$ & \begin{tabular}{|l|} 
Antifuse \\
Tech- \\
nology/ \\
CMOS \\
\end{tabular} & $\begin{array}{l}\text { H: (TAMU09DEC) } \\
\text { MB }\end{array}$ & $\begin{array}{l}\text { SEL LET } \text { th }_{\text {th }}>80 ; \\
2.5<\text { SEU LET }\end{array}$ & Y & $\begin{array}{l}1,5 \mathrm{~V} \text { Core, } \\
2.5 \mathrm{~V}, 3.3 \mathrm{~V}\end{array}$ & 2 & [15] \\
\hline $\begin{array}{l}\text { XC5VLX30T- } \\
\text { 1FFG665GU }\end{array}$ & Xilin $x$ & 0849 & $\begin{array}{l}\text { Virtex V } \\
\text { FPGA }\end{array}$ & $\begin{array}{l}65 \mathrm{~nm} \\
\text { CMOS }\end{array}$ & $\begin{array}{l}\text { H: (TAMU09SEPT) } \\
\text { MB; } \\
\text { P: (UCD09JUN) } \\
\text { MB; P: (IU09AUG) } \\
\text { MB }\end{array}$ & $\begin{array}{l}\mathrm{H}: \text { SEL LET } \\
\text { th }>75 ; \\
\text { SEU LET } \\
\text { P: SEUs observed for all } \\
\text { energies tested }(0.9,1.4,2.3, \\
5.9,7.8,13.2,21.8, \text { and } 63.8 \\
\text { MeV); SEU } \sigma=5 \times 10^{-17} \\
\mathrm{~cm}^{2} / \text { bit at } 0.9 \mathrm{MeV}^{-12} \mathrm{SEU} \sigma \text { max } \\
\text { measured }=2 \times 10^{-14} \mathrm{~cm}^{2} / \mathrm{bit} \text { at } \\
\sim 20 \mathrm{MeV} . \\
\end{array}$ & Y & $\begin{array}{l}1,2 \mathrm{~V}, 1.8 \mathrm{~V} \\
2.5 \mathrm{~V}, 3.3 \mathrm{~V}\end{array}$ & 3 & [17] \\
\hline
\end{tabular}




\begin{tabular}{|c|c|c|c|c|c|c|c|c|c|c|}
\hline Part Number & Manufacturer & LDC & $\begin{array}{l}\text { Device } \\
\text { Function }\end{array}$ & $\begin{array}{c}\text { Tech- } \\
\text { nology }\end{array}$ & $\begin{array}{c}\text { Particle: } \\
\text { (Facility/Date) P.I., }\end{array}$ & $\begin{array}{l}\text { Test Results LET in } \\
{\mathrm{MeV} \cdot \mathrm{cm}^{2} / \mathrm{mg} \sigma \text { in }} \\
\mathrm{cm}^{2} / \text { device, unless } \\
\text { otherwise specified }\end{array}$ & 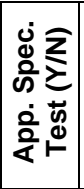 & 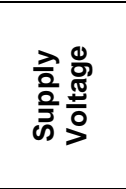 & 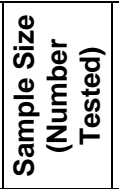 & 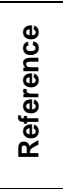 \\
\hline \multicolumn{11}{|l|}{ Linear Devices: } \\
\hline LTC6400 & $\begin{array}{c}\text { Linear } \\
\text { Technology }\end{array}$ & $\begin{array}{l}0746 \\
0705\end{array}$ & $\begin{array}{l}\text { Differential } \\
\text { Amplifier }\end{array}$ & $\begin{array}{c}\text { SiGe } \\
\text { BiCMOS }\end{array}$ & $\begin{array}{l}\text { H: (TAMU09MAY) } \\
\text { DC; } \\
\text { P: (IU09AUG) DC }\end{array}$ & \begin{tabular}{|l|} 
H: SEL LET th $>49.6 ;$ \\
SET LET \\
SET $\sigma$ increases linearly with \\
frequency. \\
SETs from $10 \mathrm{MHz}$ signals \\
are relatively minor. At 100 \\
MHz, the SETs appear \\
mostly as short duration \\
voltage spikes. At $1000 \mathrm{MHz}$, \\
the majority of SETs "erase" \\
the signal for several cycles. \\
P: SET $\sigma_{\text {max measured }} 1.5 \times 10^{-11}$ \\
$\mathrm{~cm}^{2}$ for a fluence of $1 \times 10^{12}$ \\
particles/cm², with the device \\
operating at $200 \mathrm{MHz}$. \\
No SETs were observed at \\
$10 \mathrm{MHz}$.
\end{tabular} & $\mathrm{N}$ & $3 \mathrm{~V}$ & $\begin{array}{l}2 \text { (May); } \\
1 \text { (Aug) }\end{array}$ & $\begin{array}{l}{[18]} \\
{[19]} \\
{[20]}\end{array}$ \\
\hline THS4304 & $\begin{array}{c}\text { Texas } \\
\text { Instrument }\end{array}$ & Ост04 & $\begin{array}{c}\text { Operational } \\
\text { Amplifier }\end{array}$ & $\begin{array}{c}\text { SiGe } \\
\text { BiCMOS }\end{array}$ & $\begin{array}{l}\text { H: (TAMU09AUG) } \\
\text { DC }\end{array}$ & $\begin{array}{l}\text { SET LET } \\
\text { waveforms, } 15.4 \text { for } 200 \mathrm{MHz} \\
\text { waveforms, and } 31.2 \text { for } 10 \\
\text { MHz waveforms }\end{array}$ & $\mathrm{N}$ & $2.5 \mathrm{~V}$ & 2 & $\begin{array}{l}{[20]} \\
{[21]}\end{array}$ \\
\hline \multicolumn{11}{|l|}{ Memory Devices: } \\
\hline $\begin{array}{l}\text { Part Number: } \\
\text { unavailable }\end{array}$ & $\begin{array}{c}\text { Manufacturer: } \\
\text { unavailable }\end{array}$ & $\begin{array}{l}\text { LDC and } \\
\text { die } \\
\text { markings: } \\
\text { un- } \\
\text { available }\end{array}$ & $\begin{array}{l}\text { Phase } \\
\text { Change } \\
\text { Memory } \\
\text { (PCM) }\end{array}$ & $\begin{array}{c}90 \mathrm{~nm} \\
\text { CMOS } \\
\text { Non- } \\
\text { volatile } \\
\text { Memory }\end{array}$ & $\begin{array}{l}\text { H: (TAMU09AUG) } \\
\text { HK, KL; } \\
\text { H: (TAMU09DEC) } \\
\text { HK, KL }\end{array}$ & \begin{tabular}{|l|} 
A commercial sample of a \\
90nm CMOS phase change \\
non-volatile memory was \\
tested for heavy ion SEE \\
tolerance at TAMU. Static \\
and dynamic tests were \\
performed with a variety of \\
test patterns including \\
checkerboard and inverse \\
checkerboard. Static testing \\
indicated that the phase \\
change storage cells were \\
hard to the highest tested \\
LET of 112 . No permanent \\
device failures were \\
observed. Even at elevated \\
temperature (70oC) and $V_{d d}$ \\
$+10 \%$, the device did not \\
suffer permanent failures, \\
though SEL was observed. \\
However, the device showed \\
low SEFI threshold at LET \\
below 2.9 suggesting that \\
control circuits are the weak \\
link. Though non destructive, \\
its low SEL LET th below 2.9 \\
cannot be ignored either. \\
\end{tabular} & $\mathrm{N}$ & $\begin{array}{l}2.7 ; 3.0 \\
3.6 \mathrm{~V}\end{array}$ & 4 & $\mathrm{~N} / \mathrm{A}$ \\
\hline $\begin{array}{c}\text { K4B1GO846D- } \\
\text { HCH9 }\end{array}$ & Samsung & $\begin{array}{c}0813 \\
\text { Markings: } \\
\text { SEC813H } \\
\text { CH9 } \\
\text { GNL037B } \\
\text { B }\end{array}$ & $\begin{array}{l}\text { DDR3 } \\
\text { SDRAM }\end{array}$ & $\begin{array}{l}65 \mathrm{~nm} \\
\text { CMOS }\end{array}$ & $\begin{array}{l}\text { H: (TAMU09AUG) } \\
\text { RL } \\
\text { P: (IU09AUG) RL }\end{array}$ & 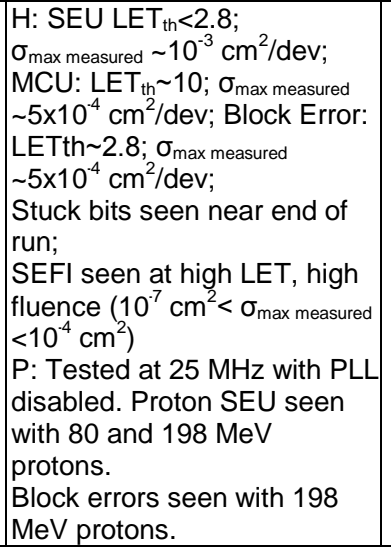 & 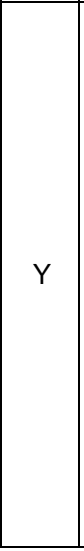 & $1.8 \mathrm{~V}$ & 2 & [22] \\
\hline
\end{tabular}




\begin{tabular}{|c|c|c|c|c|c|c|c|c|c|c|}
\hline Part Number & Manufacturer & LDC & $\begin{array}{l}\text { Device } \\
\text { Function }\end{array}$ & $\begin{array}{c}\text { Tech- } \\
\text { nology }\end{array}$ & $\begin{array}{c}\text { Particle: } \\
\text { (Facility/Date) P.I., }\end{array}$ & $\begin{array}{l}\text { Test Results LET in } \\
\mathrm{MeV} \cdot \mathrm{cm}^{2} / \mathrm{mg} \sigma \text { in } \\
\mathrm{cm}^{2} / \mathrm{device} \text {, unless } \\
\text { otherwise specified }\end{array}$ & 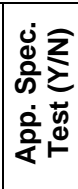 & 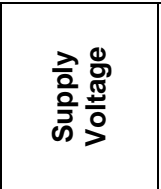 & 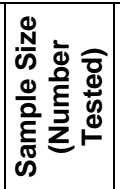 & 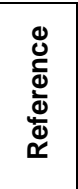 \\
\hline $\begin{array}{l}\text { K9F4G08U0A- } \\
\text { PCB0 }\end{array}$ & Samsung & $\begin{array}{l}0840 \\
0843 \\
0846 \\
0901 \\
0907\end{array}$ & \begin{tabular}{|} 
4Gbit NAND \\
Flash \\
Memory
\end{tabular} & CMOS & $\begin{array}{l}\text { H: (TAMU09MAY) } \\
\text { TO; AS } \\
\text { H: (TAMU09AUG) } \\
\text { TO w/F. Irom } \\
\text { testing LDC } 0907 \\
\text { only } \\
\end{array}$ & $\begin{array}{l}\text { Bit error } \mathrm{LET}_{\text {th }} \sim 2.8 \text {; } \\
\text { Write mode failures were } \\
\text { observed at } 70^{\circ} \mathrm{C} \text { at LET } \\
54.8 \text {. }\end{array}$ & $\mathrm{N}$ & $\begin{array}{c}3.3 \mathrm{~V} \text { for } \\
\text { SEU and } \\
\text { SEFI, 3.6V } \\
(3.3+10 \%) \\
\text { for SEL }\end{array}$ & 23 & $\begin{array}{l}{[23]} \\
{[24]}\end{array}$ \\
\hline $\begin{array}{c}\text { MT29F4G08AA } \\
\text { AWP }\end{array}$ & Micron & 0744 & \begin{tabular}{|} 
4Gbit NAND \\
Flash \\
Memory
\end{tabular} & CMOS & $\begin{array}{l}\text { H: (TAMU09AUG) } \\
\text { TO w/ F. Irom }\end{array}$ & $\begin{array}{l}31<\text { SEL LET }_{\text {th }}<54.8 ; \\
2.8<\text { destructive failure } \\
\text { (erase failure) }<8.4 \text {; Bit } \\
\text { errors were observed at 2.8; } \\
\text { SEFIs were observed at } \\
\text { LET 8.4; }\end{array}$ & $\mathrm{N}$ & $3.3 \mathrm{~V}$ & 1 & $\begin{array}{l}{[24]} \\
{[25]}\end{array}$ \\
\hline \multicolumn{11}{|l|}{ Power Devices: } \\
\hline CSD16403Q5A & $\begin{array}{l}\text { Texas } \\
\text { Instrument }\end{array}$ & $\begin{array}{l}0916 \mathrm{C} \\
6 \mathrm{~W} .08\end{array}$ & $\begin{array}{l}\text { 25V N- } \\
\text { Channel } \\
\text { Power } \\
\text { MOSFET }\end{array}$ & \begin{tabular}{|c|} 
Commer \\
cial \\
NexFET $^{\top}$ \\
$\mathrm{M}_{\mathrm{n}-}$ \\
channel \\
lat./vert. \\
hybrid \\
\end{tabular} & $\begin{array}{l}\text { H: (TAMU09DEC) } \\
\text { JML }\end{array}$ & $\begin{array}{l}\text { SEB last pass/first fail } V_{d s}= \\
13 \mathrm{~V} / 14 \mathrm{~V} \text { for } \mathrm{LET}=27(\mathrm{Kr}) \& \\
41.5(\mathrm{Ag}) \text {, at all test } \mathrm{V}_{\mathrm{gs} .}\end{array}$ & $\mathrm{N}$ & $\begin{array}{c}\text { OVgs, } \\
5 \mathrm{Vgs}, 7 \mathrm{Vgs}\end{array}$ & 18 & [26] \\
\hline IRH7360SE & $\begin{array}{c}\text { International } \\
\text { Rectifier }\end{array}$ & $\begin{array}{c}\text { No LDC. } \\
\text { Markings } \\
\text { R473288- } \\
21\end{array}$ & $\begin{array}{l}\text { Power } \\
\text { MOSFET }\end{array}$ & $\begin{array}{c}\text { Gen } 4 \mathrm{n}- \\
\text { channel } \\
\text { VDMOS } \\
\text { FET }\end{array}$ & $\begin{array}{l}\text { H: (TAMU09MAR) } \\
\text { JML }\end{array}$ & \begin{tabular}{|l}
$\mathrm{LET}=28.1$ (Kr): no failure at \\
$0 \mathrm{~V}_{\mathrm{gs}} / 400 \mathrm{~V}_{\mathrm{ds}}$; Failed at - \\
$15 \mathrm{~V}_{\mathrm{g}} / 330 \mathrm{~V}_{\mathrm{ds}}$ (last pass \\
$320 \mathrm{~V}_{\mathrm{ds}}$ ). \\
$\mathrm{LET}=41.9$ (Ag): $\mathrm{SEGR}$ at \\
$0 \mathrm{~V}_{\mathrm{gg}} / 210 \mathrm{~V}_{\mathrm{ds}}$ (last pass \\
$200 \mathrm{~V}_{\mathrm{ds}}$ ) and at $-15 \mathrm{~V}_{\mathrm{gs}} 140 \mathrm{~V}_{\mathrm{ds}}$ \\
(last pass $130 \mathrm{~V}_{\mathrm{ds}}$ ).
\end{tabular} & $\mathrm{N}$ & $\begin{array}{l}\text { OVgs; } \\
-15 \mathrm{Vgs}\end{array}$ & 11 & {$[27]$} \\
\hline ISL70001 & Intersil & $\begin{array}{c}\text { NA (Part } \\
\text { in } \\
\text { develop- } \\
\text { ment) }\end{array}$ & $\begin{array}{c}\text { POL DC/DC } \\
\text { Converter }\end{array}$ & BiCMOS & $\begin{array}{l}\text { H: (TAMU09MAY; } \\
\text { TAMU09AUG) DC } \\
\text { w/Intersil }\end{array}$ & $\begin{array}{l}\text { SEL / SEB / SEGR } \\
\text { LETth>86.4 up to } 125^{\circ} \mathrm{C}\end{array}$ & $\mathrm{N}$ & $5.7 \mathrm{~V}$ & $>10$ & [28] \\
\hline MSK5820-2.5RH & M.S. Kennedy & 0923 & $\begin{array}{l}\text { Voltage } \\
\text { Regulator }\end{array}$ & $\begin{array}{l}\text { Linear } \\
\text { Bipolar } \\
\text { Hybrid }\end{array}$ & L: (NRL09OCT) JP & $\begin{array}{l}\text { Positive and negative } \\
\text { transients were observed } \\
(-12 \text { to }+100 \mathrm{mV}) \text {, up to a } \\
\text { width of } \sim 225 \mu \mathrm{s} \text {. } \\
\end{array}$ & $\mathrm{Y}$ & $\begin{aligned} \mathrm{V}_{\text {in }} & =5 \mathrm{~V} \\
\mathrm{~V}_{\text {out }} & =2.5 \mathrm{~V}\end{aligned}$ & 1 & [29] \\
\hline MSK5900RH & M.S. Kennedy & 0703 & $\begin{array}{l}\text { Voltage } \\
\text { Regulator }\end{array}$ & $\begin{array}{l}\text { Linear } \\
\text { Bipolar } \\
\text { Hybrid }\end{array}$ & L: (NRL09OCT) JP & $\begin{array}{l}\text { Positive and negative } \\
\text { transients were observed } \\
(-20 \text { to }+40 \mathrm{mV}) \text {, up to a } \\
\text { width of } \sim 200 \mu \mathrm{s} \text {. } \\
\end{array}$ & $\mathrm{Y}$ & $\begin{aligned} V_{\text {in }} & =5 \mathrm{~V} \\
V_{\text {out }} & =2.5 \mathrm{~V}\end{aligned}$ & 1 & {$[30]$} \\
\hline TPS79133 & $\begin{array}{l}\text { Texas } \\
\text { Instrument }\end{array}$ & 0710 & $\begin{array}{l}\text { Voltage } \\
\text { Regulator }\end{array}$ & BiCMOS & L: (NRL09JUN) DC & $\begin{array}{l}\text { SET pulse includes a positive } \\
\text { and negative voltage spike, } \\
\text { with pulse amplitudes }=-0.2 \\
\text { V to } 0.2 \mathrm{~V} \text { and pulse width } \\
\text { (FWHM) } 10-20 \mu \mathrm{s} \text { for each } \\
\text { spike. Pulse amplitude and } \\
\text { width increase slightly at } \\
\text { elevated temperature (373K). }\end{array}$ & $\mathrm{N}$ & $3.3 \mathrm{~V}$ & 4 & [31] \\
\hline \multicolumn{11}{|l|}{ Test Chips: } \\
\hline Test Vehicle & IBM & $\begin{array}{c}\text { No LDC } \\
\text { (test chip) }\end{array}$ & SRAM & $\begin{array}{l}45 \mathrm{~nm} \\
\mathrm{SOI} \\
\mathrm{CMOS}\end{array}$ & $\begin{array}{l}\text { P: (UCD09JUN) JP } \\
\text { P: (IU09AUG) JP }\end{array}$ & $\begin{array}{l}\text { Completed further mapping of } \\
\text { low-energy proton sensitivity. } \\
\text { High-energy proton testing } \\
\text { was done at approximately } \\
25 \mathrm{MHz} \text { for better MCU } \\
\text { statistics. }\end{array}$ & $\mathrm{N}$ & $0.6-1.2 \mathrm{~V}$ & $\begin{array}{c}2, \text { com- } \\
\text { bined } \\
\text { from all } \\
\text { tests }\end{array}$ & [32] \\
\hline Test Vehicle & $\begin{array}{c}\text { Texas } \\
\text { Instrument }\end{array}$ & $\begin{array}{c}\text { No LDC } \\
\text { (test chip) }\end{array}$ & SRAM & $\begin{array}{c}45 \mathrm{~nm} \\
\text { bulk } \\
\text { CMOS }\end{array}$ & $\begin{array}{l}\text { P: (GSFC09MAY) } \\
\text { JP/MX } \\
\text { H: (TAMU09MAY) } \\
\text { JP/MX } \\
\text { P: (UCD09JUN) } \\
\text { JP/MX } \\
\text { P: (IU09AUG) } \\
\text { JP/MX } \\
\text { H: (LBNL09SEP) } \\
\text { JP/MX [33] }\end{array}$ & $\begin{array}{l}\text { H: SEL observed at effective } \\
\text { LETs as low as 30, though } \\
\text { portions of SRAM are hard to } \\
\text { SEL up to LET } 60 \text {. } \\
\text { P: No SEL observed with } \\
\text { 198MeV protons. }\end{array}$ & $\mathrm{N}$ & $1.2 \mathrm{~V}$ & $\begin{array}{c}6, \text { com- } \\
\text { bined } \\
\text { from all } \\
\text { tests }\end{array}$ & none \\
\hline
\end{tabular}




\begin{tabular}{|c|c|c|c|c|c|c|c|c|c|c|}
\hline Part Number & Manufacturer & LDC & $\begin{array}{l}\text { Device } \\
\text { Function }\end{array}$ & $\begin{array}{l}\text { Tech- } \\
\text { nology }\end{array}$ & \begin{tabular}{c|} 
Particle: \\
(Facility/Date) P.I.,
\end{tabular} & $\begin{array}{l}\text { Test Results LET in } \\
\mathrm{MeV} \cdot \mathrm{cm}^{2} / \mathrm{mg} \sigma \text { in } \\
\mathrm{cm}^{2} / \mathrm{device} \text {, unless } \\
\text { otherwise specified }\end{array}$ & 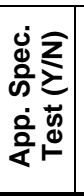 & 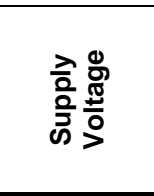 & 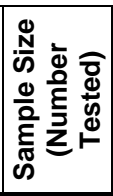 & 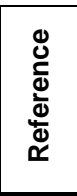 \\
\hline \multicolumn{11}{|l|}{$\begin{array}{l}\text { Miscellaneous } \\
\text { Devices: }\end{array}$} \\
\hline OV5633 & Omnivision & $\begin{array}{l}\text { No LDC } \\
\text { (image } \\
\text { sensor) }\end{array}$ & $\begin{array}{c}5 \text { megapixel } \\
\text { Image } \\
\text { Sensor }\end{array}$ & CMOS & $\begin{array}{l}\text { H: (TAMU09SEPT) } \\
\text { CM/MaC }\end{array}$ & $\begin{array}{l}\text { SEL LET } \\
\text { corrected }>87 \text {; Fluence } \\
\text { in SEFI mode. }\end{array}$ & $\mathrm{N}$ & $\begin{array}{c}\text { Core 1.5 V; } \\
\text { I/O 1.8V; } \\
\text { Analog 2.8 } \\
\text { V; Pixel 2.8 } \\
\text { V; MIPI } \\
1.5 \mathrm{~V}\end{array}$ & 4 & none \\
\hline $\begin{array}{l}\text { Part Number: } \\
\text { unavailable }\end{array}$ & $\begin{array}{c}\text { Manufacturer: } \\
\text { unavailable }\end{array}$ & $\begin{array}{l}\text { LDC and } \\
\text { die } \\
\text { markings: } \\
\text { un- } \\
\text { available }\end{array}$ & $\begin{array}{l}\text { Read Out } \\
\text { Integrated } \\
\text { Circuit } \\
\text { (ROIC) }\end{array}$ & $\begin{array}{l}0.5 \mu \mathrm{m} \\
\text { CMOS }\end{array}$ & $\begin{array}{l}\text { H: (TAMU09SEPT) } \\
\text { CM }\end{array}$ & $\begin{array}{l}\text { No SEL observed at } 40 \mathrm{~K} \text {, } \\
48 \mathrm{~K} \text { and } 80 \mathrm{~K} \text { to } \sigma_{\text {limiting }} \\
\sim 2 \times 10^{-7} \mathrm{~cm}^{2} \text { for LET }=102 ; \\
\text { Single recoverable SEL event } \\
\text { observed at } 32 \mathrm{~K} \text { to fluence }= \\
3.5 \times 10^{6} \mathrm{~cm}^{-2} \text { at } \mathrm{LET}=102 ; \\
\text { At } 20 \mathrm{~K}, \mathrm{ROIC} \text { sensitive to } \\
\mathrm{SEL} \text { for } 12=\mathrm{LET}<102 \text { with } \\
2.0 \times 10^{-5}<\sigma<3.7 \times 10^{-4} \mathrm{~cm}^{2} \\
\text { (lowest test LET). At } 300 \mathrm{~K} \text {, } \\
\mathrm{SEL} \sigma \sim 1 \times 10^{-3} \mathrm{~cm}^{2} \text { at LET }= \\
87\end{array}$ & $\mathrm{~N}$ & $5.7 \mathrm{~V}$ & 2 & {$[34]$} \\
\hline
\end{tabular}

\section{TEST RESULTS AND DISCUSSION}

As in our past workshop compendia of GSFC test results, each DUT has a detailed test report available online at http://radhome.gsfc.nasa.gov [11] describing in further detail the test method, SEE conditions/parameters, test results, and graphs of data.

This section contains summaries of testing performed on a selection of featured parts.

\section{A. Texas Instruments ADS5483 ADC}

This study was undertaken to determine the Single Event Effects (SEE), susceptibility of the Texas Instruments (TI) ADC converter: ADS5483. The DUTs were evaluated with heavy ions and protons.

There was a total of 7 ADC tested. 2 were made available for heavy ion testing, including 1 control sample. 4 devices were made available for proton testing. A TI Evaluation board with one embedded ADC (DUT) was used as a daughter card that was physically connected to the NASA Goddard low cost digital tester (LCDT). The TI Evaluation board number is ADS548xEVM. The identification information for these ADCs is as follows:

Test Chip: ADS5483

Lot \# unknown - delidded by TI

Markings:

AZ5483

TI $83 \mathrm{~K}$

E7N0 G

The DUT technology is Texas Instruments complementary bipolar process BiCom3x. The following are some of the ADS5483 Features (please refer to the ADS5483 datasheet for a complete description):

-16-bit resolution. 78 dBFS Noise Floor

-170MSPS Sample Rate

- $\mathrm{SFDR}=95 \mathrm{dBc}$
- On-Chip High Impedance Analog Buffer

- Efficient DDR LVDS-Compatible Outputs

- Power-Down Mode: 70mW

- Industrial Temperature Range: $-40^{\circ} \mathrm{C}$ to $85^{\circ} \mathrm{C}$

- 3 Vpp Differential Input Range

- $5 \mathrm{~V}$ or $3.3 \mathrm{~V}$ Power supply

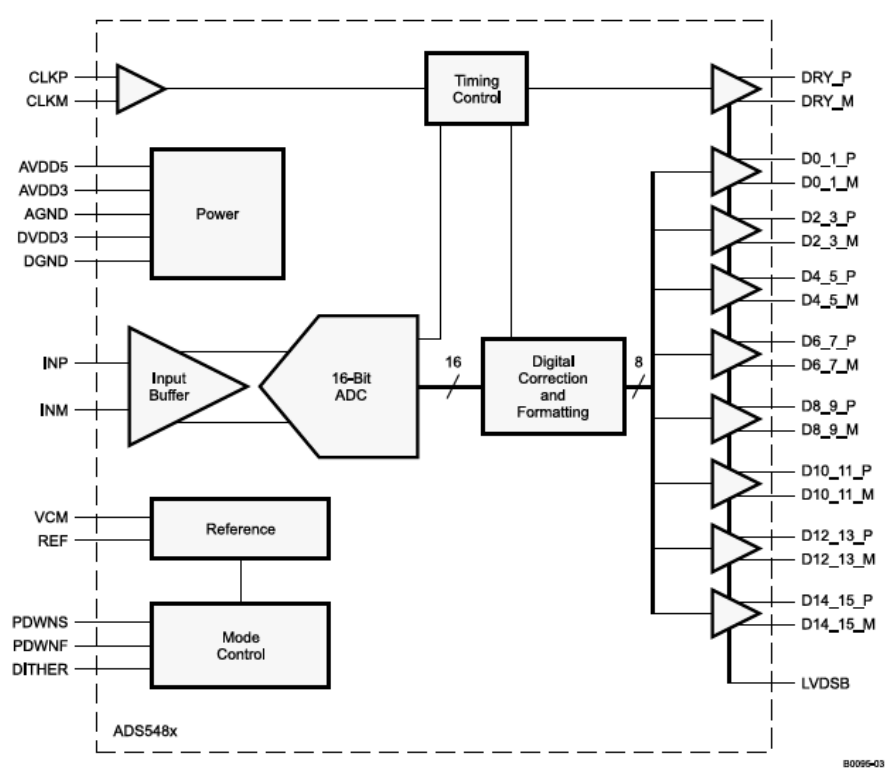

Fig. 1. Functional Block Diagram of the ADS5483.

For reliable SEU response analysis, it is important to filter out the non-SEU noise that is introduced from both the test vehicle and the ADC device. Subsequently, prior to radiation testing, system noise was measured. A minimal error-bound (EB) was calculated per test set-up such that no ADC output code errors existed during operation and pre-irradiation. The EB code value can be translated to its corresponding voltage level $\left(\mathrm{V}_{\mathrm{EB}}\right)$ as illustrated in (1). 


$$
V_{E B}=\frac{E B^{*} V p p}{2^{N b}}
$$

Concerning (1), $\mathrm{Nb}$ is the number of ADC output bits (16bits for the ADS5483) and $\mathrm{V}_{\mathrm{pp}}$ is the peak-to-peak manufacturer supplied voltage range $\left(3 \mathrm{~V}_{\mathrm{pp}}\right.$ for the ADS5483).

Regarding heavy ion testing, the ADS5483 DUT was tested at the TAMU using a $15 \mathrm{MeV} / \mathrm{amu}$ tune at room temperature. All tests were run with $10^{3}<$ flux rate $<10^{4}$ particles $/ \mathrm{cm}^{2} / \mathrm{s}$. Effective LETs ranged from $2.5 \mathrm{MeV} \cdot \mathrm{cm}^{2} / \mathrm{mg}$ to $83.4 \mathrm{MeV} \cdot \mathrm{cm}^{2} / \mathrm{mg}$ by varying the ion and by varying the angle of incidence. All proton tests were performed at IUCF. Data was obtained for two proton energies: $78 \mathrm{Mev}$ and $198 \mathrm{MeV}$. The ADC devices were operated at nominal room temperature using active cooling. A function generator was utilized as the clock and data input to the ADC. Measurements were taken by interfacing the ADC 16-bit digital outputs to the LCDT. The LCDT processed the output data and reported errors to the user host computer.

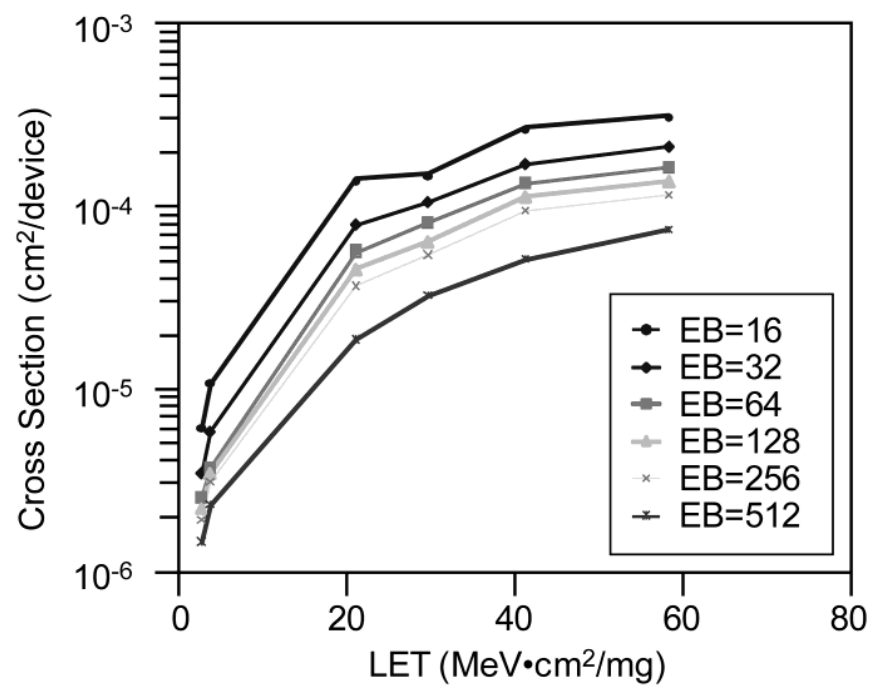

Fig. 2. Heavy Ion SEU Error Cross Sections.

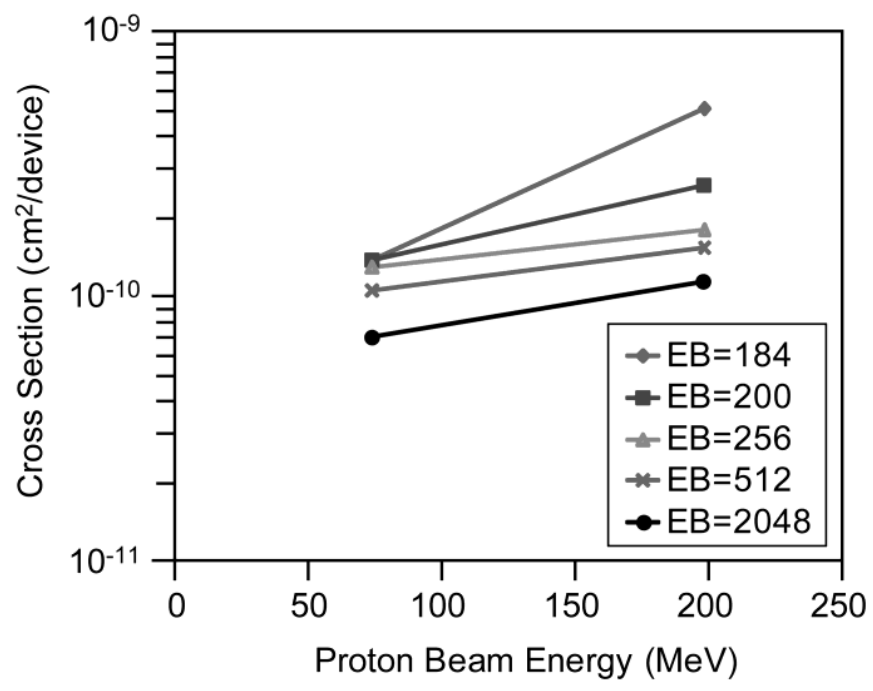

Fig. 3. Proton SEU Error Cross Sections.
As a summary, the SEU/SET response of the ADS5483 consists of:

1. Code upsets that only last for one ADC clock cycle.

2. Code upsets that last for multiple ADC clock cycles (bursts).

a. Heavy ion bursts were not as frequent as proton bursts. The longest heavy ion burst lasted for 39 ADC clock cycles.

b. Bursts due to $198 \mathrm{MeV}$ proton strikes could last thousands of ADC clock cycles. However, at $\mathrm{EB}=7.8 \mathrm{mV}$ and above, the burst duration and frequency is significantly reduced. This is due to the fact that most of the burst upsets were small upsets from the expected values - i.e. the errors jittered around the expected values. This suggests that the analog circuitry has a significant sensitivity to $198 \mathrm{MeV}$ protons.

c. Most upsets due to $73 \mathrm{MeV}$ protons were single ADC clock cycle upsets.

3. No clock losses were observed - however, this is still under investigation.

4. SEU/SET rate did not increase significantly with frequency (10 MHz vs. $100 \mathrm{MHz}$ ) [13], [14].

\section{B. Actel RTAX2000S RTAX-S FPGA EDAC Memory Tests}

This study was undertaken to determine the single event destructive and transient susceptibility of the internal memory structures embedded in the RTAX-S FPGA family of devices. The DUTs were configured to have various forms of active embedded memory structures. The memory and its supportive circuitry were monitored for SET and SEU induced faults by exposing them to a heavy ion beam. The purpose of this RTAX-S memory evaluation was to enhance prior testing of the device. The study included both static and dynamic modes of memory control and operation for memory configurations that utilized the embedded EDAC circuitry provided by Actel versus memory configurations that did not contain EDAC. It is important to note that the embedded EDAC circuitry does not reside in the hardened by design user-cells of the RTAX. Therefore, the embedded circuitry is assumed to be more susceptible than custom user designed EDAC that would utilize the RTAX-S hardened cells.

There was one RTAX-S device that contained the memory configuration under evaluation. The sample size per device (in this case) was not the focus since they are production- high speed parts with very little variation across the CMOS process. The emphasis was to test variations over the design state space. The devices were manufactured on an advanced $0.15 \mu \mathrm{m}$ CMOS Antifuse Process Technology with 7 layers of metal. The manufacturer is Actel. The devices tested had LDC of 0526 . 


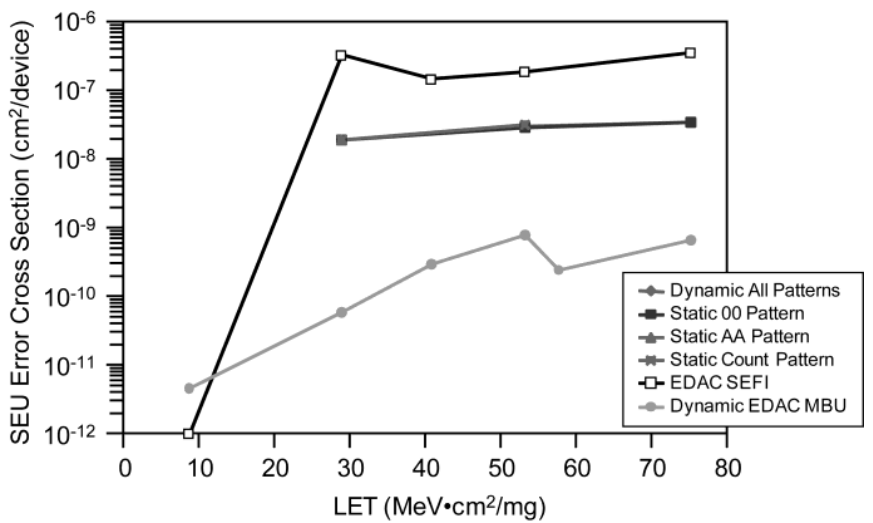

Fig. 4. SEU Error Cross Sections. Static and dynamic memory reads were evaluated for several patterns for memory structures with and without EDAC controls.

Fig. 4 illustrates that the memory bit-cell SEU cross sections over all data patterns were statistically equivalent. Dynamic tests consisted of read-write-modify cycles. Regarding memory cell SEU-cross sections, there was no statistical difference between dynamic memory operations during DUT irradiation versus static memory operation. With dynamic testing, multiple bit failures (MBUs) per address did occur at $8.5 \mathrm{MeV} \cdot \mathrm{cm}^{2} / \mathrm{mg}$. Because the EDAC structures are Single Error Correct Double Error Detect (SECDED), memory reads that utilized the embedded EDAC structures had failures. No testing was performed below $8.5 \mathrm{MeV} \cdot \mathrm{cm}^{2} / \mathrm{mg}$, therefore no on-set for memory MBU and EDAC failure has been determined. For LET $>20 \mathrm{MeV} \cdot \mathrm{cm}^{2} / \mathrm{mg}$, EDAC SEFI's also occurred. An EDAC SEFI pertains to the event of the EDAC circuitry becoming stuck in a state where it cannot correct data and eventually corrupts good data. EDAC SEFIs at LETs $>20 \mathrm{MeV} \cdot \mathrm{cm}^{2} / \mathrm{mg}$ were significant and can be avoided by the user creating a custom EDAC with the RTAX-S user fabric [15]. Note, also see J. George, et al., [16].

\section{Linear Technology LTC6400 Differential Output Amplifier/ADC Driver}

The LTC6400 is fabricated with the JAZZ-TOWER $0.35 \mu \mathrm{m}$ $\mathrm{SiGe}$ BiCMOS process. Two parts were irradiated at room temperature with the devices operating at $\mathrm{V}_{\mathrm{CC}}=3 \mathrm{~V}, \mathrm{~V}_{\mathrm{CM}}=$ $1.25 \mathrm{~V}$, sine wave inputs of $140 \mathrm{mV}_{\mathrm{pp}}$ (large signal) or $2 \mathrm{mV}_{\mathrm{pp}}$ (small signal), at frequencies of 10, 100, and $1000 \mathrm{MHz}$. Two ion species were used for this experiment: $\mathrm{Ar}$ and $\mathrm{Kr}$.

Fig. 5 shows the SET cross-sections from large signals at 10, 100, and $1000 \mathrm{MHz}$. We observed SETs down to the lowest LET of $7.4 \mathrm{MeV} \cdot \mathrm{cm}^{2} / \mathrm{mg}$ for all frequencies of operation. The SET cross-sections increase linearly with increasing frequency. The worst case transients occur at 1000 $\mathrm{MHz}$, where several cycles of the signal are erased from an SET. Fig. 6 shows an SET at $1000 \mathrm{MHz}$. No latchup events were observed. The supply current values remained relatively unchanged, at $\sim 90 \mathrm{~mA}$, throughout irradiation.
Additionally, we found that the LTC6400 was robust against high energy protons, with relatively low SET crosssections. We irradiated 2 parts with $198 \mathrm{MeV}$ protons. The increase in SET error cross-sections from $0^{\circ}$ to $60^{\circ}$ for DUT3 is unlikely due to angular effects that result from nuclear reactions, since the final $0^{\circ}$ incident run produced a larger error cross-section than the initial normal incident run. We irradiated DUT4 at normal incidence to examine the effects of total dose and displacement damage on SET sensitivity. The significant scatter in the dataset suggests that the SET crosssections more likely follow a Poisson distribution, and do not correlate strongly with accumulated dose. The different lot date codes of the parts may also contribute to the relatively large difference in the magnitudes of SET cross-sections.

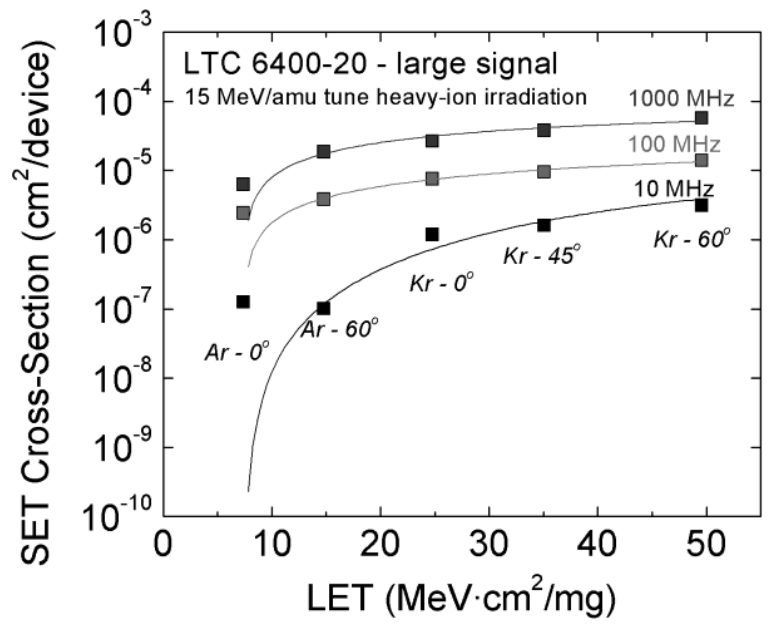

Fig. 5. Heavy-ion-induced SET error cross-sections for large signals at 10, $100 \mathrm{MHz}$, and $1 \mathrm{GHz}$.

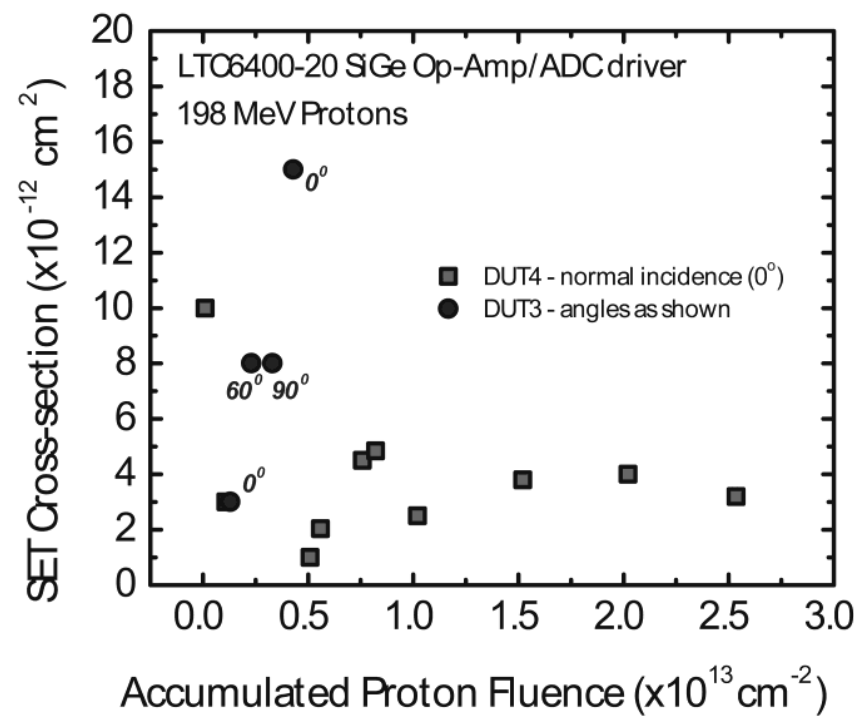

Fig. 6. SET cross section vs. accumulated dose for the LTC6400 operating at $200 \mathrm{MHz}$, irradiated with $198 \mathrm{MeV}$ protons to a fluence of $1 \times 10^{12}$ particles $/ \mathrm{cm}^{2}$. 


\section{Texas Instruments CSD16403QA Commercial Power MOSFET}

This study was undertaken to determine the single event gate rupture (SEGR) and burnout (SEB) susceptibility of the commercial CSD16403QA power MOSFET under heavy ion irradiation. The device is a $100 \mathrm{amp}, 25$ volt $\mathrm{n}$-channel power MOSFET, manufactured under Texas Instruments' recently acquired CICLON NexFET ${ }^{\mathrm{TM}}$ commercial process technology. The NexFET technology is a hybrid lateralvertical power MOSFET (Fig. 7) featuring a lateral channel under a planar gate connecting the source to the lightly doped drain extension region (LDD), and a highly doped vertical drain "sinker" that brings the current flow vertically down to the backside drain contact. We believe that this test is the first to evaluate the heavy-ion response for this device type.

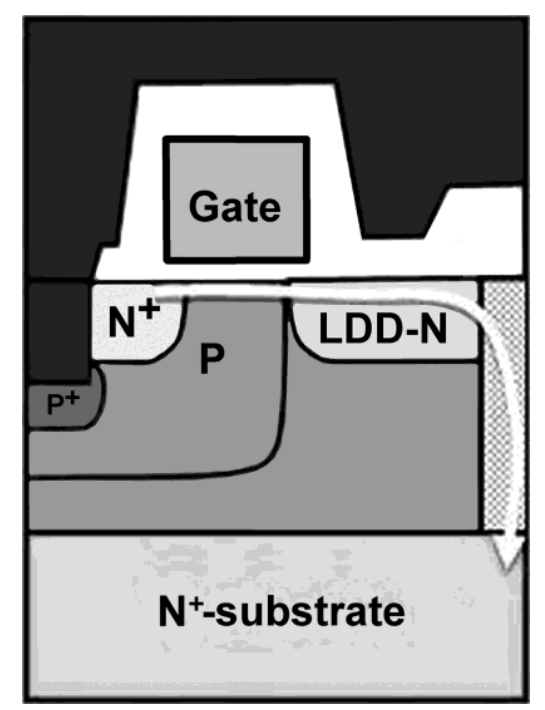

Fig. 7. Cartoon showing the structure of a n-type NexFET ${ }^{\mathrm{TM}}$ power MOSFET. From: Electronics Design, Strategy, News, 12 Feb 2009.

Tests were conducted at normal incidence to the surface. Each device was biased at one of three gate-source voltage (Vgs) biases, and the drain-source voltage (Vds) was incremented between beam runs until device failure was observed. Currents were monitored at the gate and drain nodes during testing, and voltage transients were recorded across a small sense-resistor placed behind the drain stiffening capacitor. All device failures occurred during irradiation and were due to single event burnout, although in each instance the gate ruptured. As can be seen in Fig. 8, the SEE safe operating area is $52 \%$ of the maximum rated drain voltage, independent of the gate bias applied during testing. This gatevoltage independence is a hallmark of single-event burnout. In addition, both krypton at an incident LET of $27.4 \mathrm{MeV} \cdot \mathrm{cm}^{2} / \mathrm{mg}$ and silver at an incident LET of $40.5 \mathrm{MeV} \cdot \mathrm{cm}^{2} / \mathrm{mg}$ yielded the same SEE response curves.

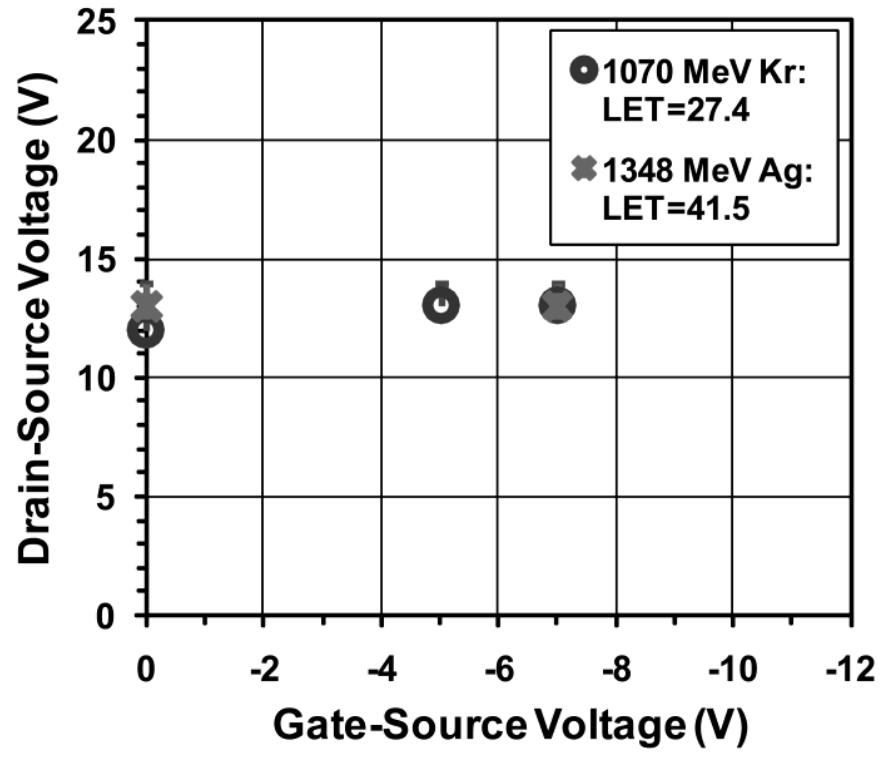

Fig. 8. SEE response curve.

Efforts to evaluate a p-channel NexFET were inconclusive due to the presence of an irremovable heat sink on top of the die obstructing more than $80 \%$ of the active region. As pchannel power MOSFETs do not suffer SEB, additional tests will reveal whether these commercial p-type structures will be naturally rugged under a heavy-ion space environment. [26]

\section{E. M.S. Kennedy Voltage Regulators MSK5900RH and MSK5820-2.5RH}

We undertook this study to characterize the applicationspecific SET behavior of the MSK5900RH and MSK58202.5RH low dropout voltage regulators using pulsed laser irradiation. Both regulators are hybrid integrated circuits, which contain controller circuitry that governs a power PNP bipolar transistor. The controller circuitry, manufactured by Linear Technology, is the LT1573 in the case of the MSK5900 and the RH1573K in the case of the MSK5820. The LT1573/RH1573 is a low dropout PNP regulator driver. The RH1573K uses the same mask set as the LT1573, but has a different passivation to improve its total ionizing dose response. 


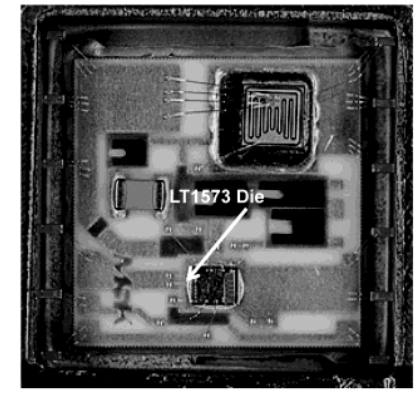

(a) MSK5900RH Package

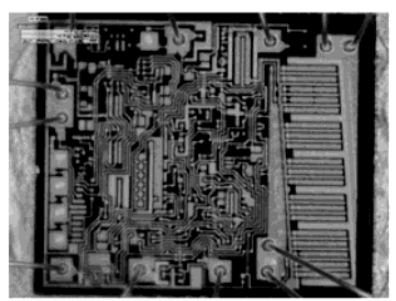

(c) Linear Technology LT1573 Die

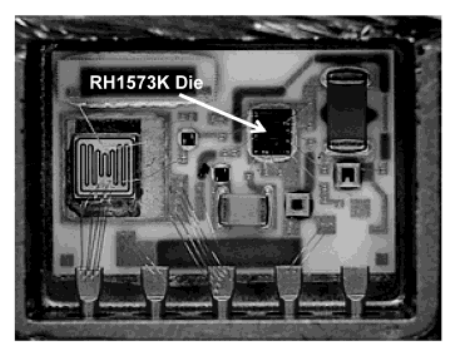

(b) MSK5820-2.5RH Package

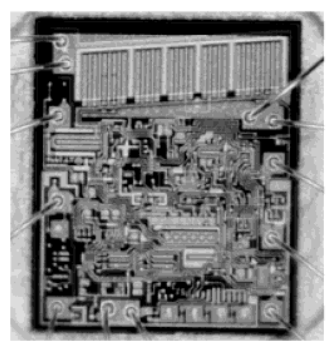

(d) Linear Technology $\mathrm{RH} 1573 \mathrm{~K}$ Die

Fig. 9. Micrographs of the MSK5900RH and MSK5820-2.5RH bipolar hybrid integrated circuits and the PNP driver regulator dice within. Note that (c) and (d) have been rotated so that their orientation is the same as in (a) and (b). The views in (c) and (d) are the same as what the laser sees through the 100x objective.

Package and die micrographs of the MSK5900RH/LT1573 and MSK5820-2.5RH/RH1573 are shown in Fig. 9. The MSK5900RH is packaged in a 12-pin flatpack and the MSK5820-2.5RH is packaged in a 5-pin single-inline package. It is clear from Figs. 9(c) and (d) that the LT1573 and RH1573 have the same mask set. The main difference is that the MSK5900RH is a positive adjustable regulator tuned with an external resistor network and the MSK5820-2.5RH is a fixed positive voltage regulator. Both regulators were configured with a $5.0 \mathrm{~V}$ input and $\mathrm{a}+2.5 \mathrm{~V}$ output.

We used the pulsed laser facility at the Naval Research Laboratory to perform single-photon absorption on the M.S. Kennedy voltage regulators with a $590 \mathrm{~nm}$ wavelength beam. The pulse energy for these experiments was in excess of an equivalent LET of $100 \mathrm{MeV} \cdot \mathrm{cm}^{2} / \mathrm{mg}$.

We scanned the laser spot across the entire surface of both the LT1573 in the MSK5900RH and the RH1573K in the MSK5820-2.5RH. Two load conditions were used for each component. The MSK5900RH was irradiated with $25 \mathrm{~mA}$ and $138 \mathrm{~mA}$ loads while the MSK5820-2.5RH was irradiated with $0.515 \mathrm{~A}$ and $2.45 \mathrm{~A}$ loads. Voltage transients were observed with all load conditions on each component. We observed both positive and negative transients $(-20$ to $+40 \mathrm{mV})$, up to a width of approximately $200 \mu \mathrm{s}$ on the MSK5900RH. The MSK5820-2.5RH also showed both positive and negative transients $(-12$ to $+100 \mathrm{mV})$, up to a width of approximately $225 \mu \mathrm{s}$. These results are shown graphically in Fig. 10 and Fig. 11 [29] [30].

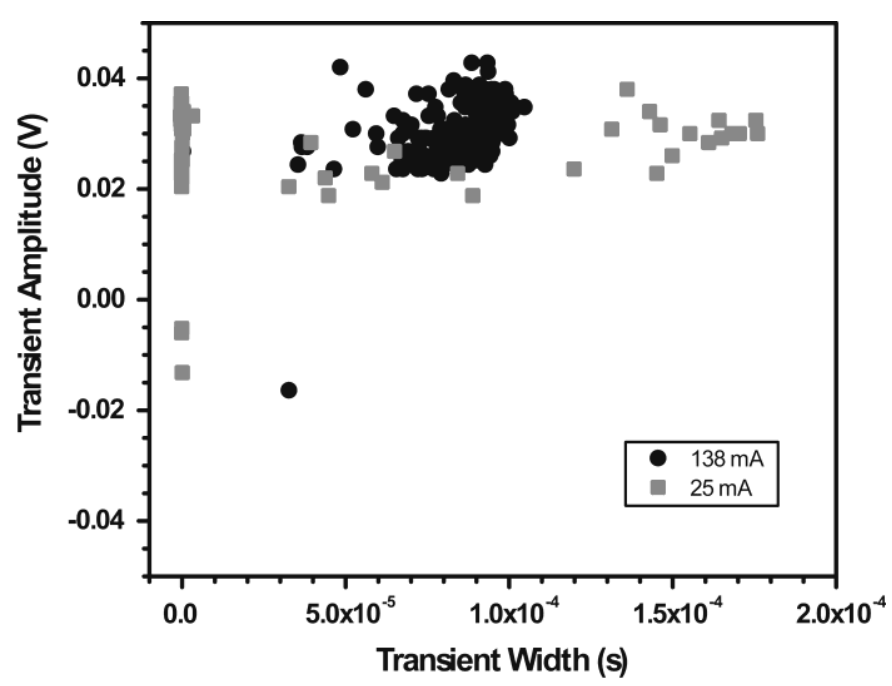

Fig. 10. MSK5900RH transients.

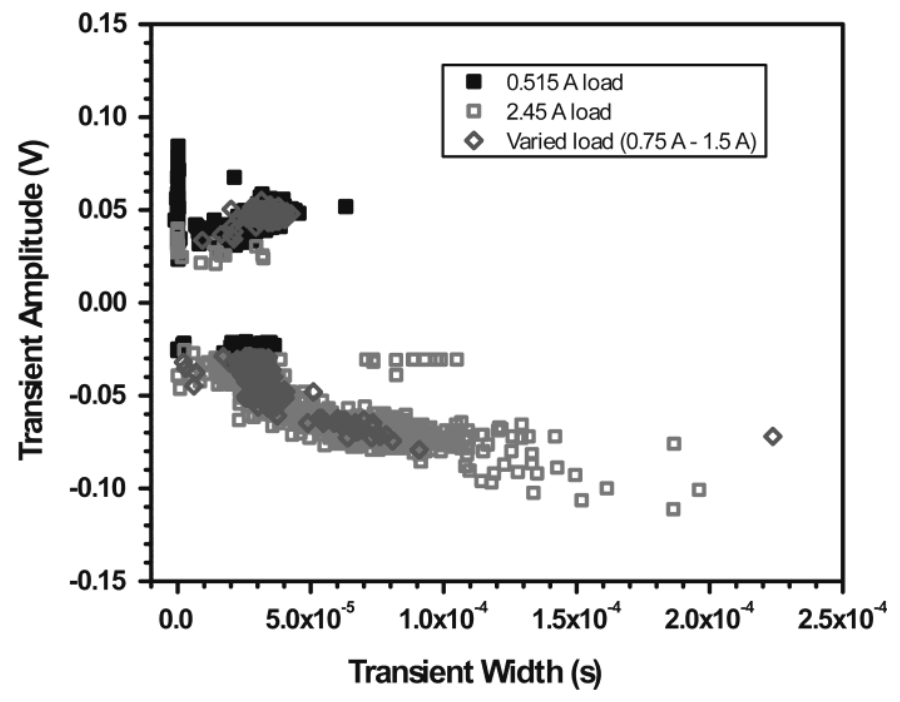

Fig. 11. MSK5820-2.5RH transients.

\section{F. Intersil ISL70001 Point-of-load DC/DC Converter}

The ISL70001SRH is a new point-of-load (POL) DC/DC converter developed by Intersil Coporation. The part has been qualified for total ionizing dose irradiation. Here we give a short summary of the single event effects performance from heavy-ion irradiations. Details of the part's radiation performance can be found in Intersil's publication. [28]

The ISL70001SRH was found to be free of SEL/SEB/SEGR up to an LET of $86.4 \mathrm{MeV} \cdot \mathrm{cm}^{2} / \mathrm{mg}$, with the device operating at an input voltage of $5.7 \mathrm{~V}$, output current of $7 \mathrm{~A}$, output voltage of $1.8 \mathrm{~V}$, and case temperature up to $125^{\circ} \mathrm{C}$.

The redundant PWM loop design of the ISL70001SRH is effective in limiting SETs. The worst case SET at an LET of $86.4 \mathrm{MeV} \cdot \mathrm{cm}^{2} / \mathrm{mg}$ was found to cause less than $1 \%$ change in the output voltage. 
A SEFI phenomenon of the Softstart function was observed at an LET of $84.6 \mathrm{MeV} \cdot \mathrm{cm}^{2} / \mathrm{mg}$ and $3 \mathrm{~V}$ input voltage only. The ISL70001SRH shuts down and then restarts normally through the Softstart function. The estimated cross section for this phenomenon is $1.4 \times 10^{-6} \mathrm{~cm}^{2}$ at an LET of $84.6 \mathrm{MeV} \cdot \mathrm{cm}^{2} / \mathrm{mg}$ and $3 \mathrm{~V}$ input. With a $5 \mathrm{~V}$ input, the SEFI cross section increased to $\sim 6.5 \times 10^{-8} \mathrm{~cm}^{2}$. [28]

\section{SUMMARY}

We have presented current data from SEE testing on a variety of mainly commercial devices. It is the authors' recommendation that this data be used with caution. We also highly recommend that lot testing be performed on any suspect or commercial device.

\section{ACKNOWLEDGMENT}

The authors gratefully thank members of the Radiation Effects and Analysis Group who contributed to the test results presented here: Mark R. Friendlich, Megan C Casey, Michael J. Campola, Donald K. Hawkins, James D. Forney, Timothy L. Irwin, Christina M. Seidleck, Stephen R. Cox, Christopher Perez, and Joseph W. Portner. The authors would also like to thank Dale McMorrow for his expert help with laser testing at NRL, Kirby Kruckmeyer for his help with supplying test devices and test boards for the ADC14155, and Duc Nguyen for his help with the testing at TAMU in August 2009 of the Samsung K9F4G08U0A-PCB0 and the Micron MT29F4G08AA AWP.

\section{REFERENCES}

[1] Kenneth A. LaBel, Lewis M. Cohn, and Ray Ladbury,"Are Current SEE Test Procedures Adequate for Modern Devices and Electronics Technologies?," http://radhome.gsfc.nasa.gov/ radhome/ papers/ HEART08_LaBel.pdf

[2] Donna J. Cochran, Dakai Chen, Timothy Oldham, Hak Kim, Stephen Buchner, Kenneth A. LaBel, Jonathan Pellish, Martin A. Carts, Martha V. O'Bryan, and Anthony B. Sanders, " Current Total Ionizing Dose and Displacement Damage Compendium of Candidate Spacecraft Electronics for NASA," submitted for publication in IEEE Radiation Effects Data Workshop, Jul. 2010.

[3] Lawrence Berkeley National Laboratory (LBNL), 88-Inch Cyclotron Accelerator Facility, http://cyclotron.lbl.gov/index.html, May 2007.

[4] B. Hyman, "Texas A\&M University Cyclotron Institute, K500 Superconducting Cyclotron Facility," http://cyclotron.tamu.edu/ facilities.htm, Jul. 2003.

[5] W.J. Stapor, "Single-Event Effects Qualification," IEEE NSREC95 Short Course, sec. II, pp 1-68, Jul. 1995.

[6] C. M. Castaneda, "Crocker Nuclear Laboratory (CNL) Radiation Effects Measurement and Test Facility," IEEE NSREC01 Data Workshop, pp. 77-81, Jul. 2001.

[7] C. C. Foster, S. L. Casey, P. Miesle, N. Sifri, A. H. Skees, K. M. Murray, "Opportunities for Single Event and Other Radiation Effects Testing and Research at the Indiana University Cyclotron Facility," IEEE NSREC96 Data Workshop, pp. 84-87, Jul. 1996.

[8] J. S. Melinger, S. Buchner, D. McMorrow, T. R. Weatherford, A. B. Campbell, and H. Eisen, "Critical evaluation of the pulsed laser method for single event effects testing and fundamental studies," IEEE Trans. Nucl. Sci., vol 41, pp. 2574-2584, Dec. 1994.

[9] D. McMorrow, J. S. Melinger, and S. Buchner, "Application of a Pulsed Laser for Evaluation and Optimization of SEU-Hard Designs," IEEE Trans. Nucl. Sci., vol 47, no. 3, pp. 559-565, Jun. 2000.

[10] R. Koga and W. A. Kolasinski, "Heavy Ion-Induced Single Event Upsets of Microcircuits; A Summary of the Aerospace Corporation Test Data," IEEE Trans. Nucl. Sci., Vol. 31, No. 6, pp. 1190 - 1195, Dec. 1984.
[11] NASA/GSFC Radiation Effects and Analysis home page, http://radhome.gsfc.nasa.gov

[12] Stephen Buchner, Melanie Berg, and Christina Seidleck, "Single Event Effects Testing of ADC14155WG-MLS Analog-to-Digital Converter (National Semiconductor)," http://radhome.gsfc.nasa.gov/ radhome/ papers/ D062209_AD14155.pdf, Jun 2009.

[13] M. Berg, S. Buchner, H. Kim, M. Friendlich, C. Perez, A. Phan, C. Seidleck, K. LaBel, and K. Kruckmeyer, "Enhancing Observability of Signal Composition and Error Signatures during Dynamic SEE Analog to Digital Device Testing," to be published in RADiation Effects on Components \& Systems (RADECS) 2009, Brugge, Belgium, Aug. 2010.

[14] Melanie Berg, Hak Kim, and Anthony Phan, "Heavy Ion SEE Tests for Texas Instruments ADS5483 ADC," http://radhome.gsfc.nasa.gov/ radhome/ papers/ T052309_ IU081009__ADS5483.pdf, May 2009.

[15] Melanie Berg, Hak Kim, Mark Friendlich, and Christopher Perez, "Heavy Ion SEE Tests for Actel RTAX2000SX/SL http://radhome.gsfc. nasa.gov/ radhome/ papers/ T110909_RTAX2000SX, Nov. 2009.

[16] Jeffrey S. George, Rocky Koga, and Mark Zakrzewski, "Single Event Effects Tests on the Actel RTAX2000S FPGA," IEEE Radiation Effects Data Workshop, pp. 140-147, Jul. 2009.

[17] Melanie Berg, "Proton SEE Tests for Xilinx Virtex V XC5VLX30T1FFG665GU FPGA," http://radhome.gsfc.nasa.gov/ radhome/ papers/ D062209_XCVLX30T, Jun. 2009.

[18] Dakai Chen and Hak Kim, "Test Report of Heavy-ion SEEs for the LTC6400-20 Differential ADC Driver," http://radhome.gsfc.nasa.gov/ radhome/ papers/ T052309_LTC6400.pdf, May 2009.

[19] Dakai Chen, Anthony Phan, and Jonathan Pellish, "Proton Irradiation Test Report for the LTC6400-20 Differential ADC Driver," http://radhome.gsfc.nasa.gov/ radhome/ papers/ I080409_LTC6400.pdf, Aug 2009.

[20] D. Chen, A. Phan, H. Kim, J. Pellish, K. LaBel,S. Burns, R. Albarian, B. Holcombe, B. Little, J. Salzman, "Radiation Performance of Commercial SiGe HBT BiCMOS High Speed Operational Amplifiers," submitted for publication in IEEE Radiation Effects Data Workshop, Jul. 2010.

[21] Dakai Chen and Anthony Phan, "Heavy-ion SEE at Elevated Temperatures Test Report for the THS4304 Wideband Operational Amplifier," http://radhome.gsfc.nasa.gov/ radhome/ papers/ T082409_THS4304.pdf, Aug 2009.

[22] Ray Ladbury, H. Kim, M. Friendlich, "DDR3 SDRAM SEE Test Report," http://radhome.gsfc.nasa.gov/ radhome/ papers/ I081009_ T082409_K4B1GO846DHCF8, Aug. 2009.

[23] Tim Oldham, Mark Friendlich, Anthony B. Sanders, Hak Kim, and Melanie Berg, "Heavy Ion SEE Test Report for the Samsung 4Gbit NAND Flash Memory for MMS,", http://radhome.gsfc.nasa.gov/ radhome/ papers/ T052309_K9F4G08U0A, May 2009.

[24] T.R. Oldham, M.R. Friendlich, D. Nguyen, and F. Irom, "Heavy Ion SEE Test of Micron and Samsung 4G NAND Flash Memories: Current Spike Experiment with JPL," http://radhome.gsfc.nasa.gov/ radhome/ papers/ T082409_K9F4G08U0_MT29F4G08AA, Aug. 2009.

[25] Tim Oldham, Mark Friendlich, Anthony B. Sanders, Hak Kim, and Melanie Berg, "Heavy Ion SEE Test Report for the Micron 4Gbit NAND Flash Memory for MMS," http://radhome.gsfc.nasa.gov/, T110308_MT29F4G08AAAWP.pdf, Nov. 2008.

[26] Jean-Marie Lauenstein, Anthony Phan, Hak Kim, and Tim Irwin, "Single event effects testing of the Texas Instruments Commercial CSD16403Q5A Power nMOSFET," http://radhome.gsfc.nasa.gov/ radhome/ papers/ T110909_CSD16403Q5A, Nov. 2009.

[27] Jean-Marie Lauenstein, "Heavy Ion Testing of the International IRH7360SE Rectifier Power MOSFET," http://radhome.gsfc.nasa.gov/ radhome/ papers/ T030409_IRH7360SE, Mar. 2009.

[28] N. W. van Vonno, L. W. Pearce, H. W. Satterfield, E. T. Thomson, A. P. Williams, T. E. Fobes, P. J. Chesley and J. S. Gill, "Total Dose and Single Event Testing of a Hardened Point of Load Regulator," submitted for publication in IEEE Radiation Effects Data Workshop, Jul. 2010.

[29] J. A. Pellish, S. Buchner, D. A. Batchelor, J. D. Forney, and C. M. Seidleck, "Pulsed Laser Single-Event Transient Testing of the M.S. Kennedy 5820-2.5RH Radiation Hardened ULDO Fixed Positive Linear Voltage Regulator," http://radhome.gsfc.nasa.gov/ radhome/ papers/ NRL102709_MSK5820.pdf, Oct 2009.

[30] J. A. Pellish, S. Buchner, D. A. Batchelor, J. D. Forney, and C. M. Seidleck, "Pulsed Laser Single-Event Transient Testing of the M.S. Kennedy 5900RH Radiation Hardened ULDO Adjustable Positive Linear Voltage Regulator," http://radhome.gsfc.nasa.gov/ radhome/ papers/ NRL102709_MSK5900RH.pdf, Oct 2009. 
[31] Dakai Chen, "Test Report for Elevated Temperature Laser Testing of the Texas Instrument TPS79133 Voltage Regulator," http://radhome. gsfc.nasa.gov/ radhome/ papers/ NRL062409_TPS79133, Jun. 2009.

[32] D. F. Heidel, P. W. Marshall, J. A. Pellish, K. P. Rodbell, K. A. LaBel, J. R. Schwank, S. E. Rauch, M. Hakey, M. D. Berg, C. Castaneda, P. E. Dodd, M. R. Friendlich, A. D. Phan, C. M. Seidleck, M. R. Shaneyfelt, and M. A. Xapsos, "Single-Event Upsets and Multiple-Bit Upsets on a 45 nm SOI SRAM," IEEE Trans. Nucl. Sci., Vol. 56, No. 6, pp. 3499 3504, Dec. 2009.

[33] N. A. Dodds, R. A. Reed, R. D. Schrimpf, L. W. Massengill, R. A. Weller, J. M. Hutson, J. A. Pellish, M. A. Xapsos, H. S. Kim, M. D. Berg, M. R. Friendlich, A. M. Phan, C. M. Seidleck, X. Deng, R. C. Baumann, "Optimization of Well and Substrate Contact Spacing for Single Event Latchup Hardness," submitted for publication in IEEE Trans. Nucl. Sci., Dec. 2010.

[34] Cheryl J. Marshall, Paul W. Marshall, Raymond L. Ladbury, Augustyn Waczynski, Roger D. Foltz, Rajan Arora, Nathaniel A. Dodds, John D. Cressler, Jonatha A. Pellish, Dakai Chen, Duncan M. Kahle, Gregory S. Delo, Emily Kan, Nicholas Boehm, Robert A. Reed, and Kenneth A. LaBel, "New Particle-Induced Single Event Latchup Mechanism Observed in a Cryogenic CMOS Readout Integrated Circuit," submitted for publication in IEEE Trans. Nucl. Sci., Dec. 2010. 